\title{
FORMATION OF LAYERED FRACTURE AND OUTBURST BY PERCOLATION
}

\author{
X.B. Lu*, X.H. Zhang, and S.Y. Wang \\ Institute of Mechanics, Chinese Academy Sciences \\ *e-mail: xblu@imech.ac.ac
}

\begin{abstract}
Summary. Formation mechanism of layered fracture and outburst in soils is investigated. It is shown that once the pore pressure is high enough to cause the overcap failure, layered fracture or outburst occurs in liquefied or gasified sediments. The formation conditions are related to the strength of sediments and the boundary friction.
\end{abstract}

Keywords: gas hydrate, layered fracture, outburst.

\section{INTRODUCTION}

Layered fracture and outburst in soil sediment can occur in many cases, for example, during liquefaction of sand (also called water film or soil boiling) [1,2], during mining of coal, and during dissociation of gas hydrate [3,4].

Layered fracture in soils is a water or gas gap which forms when the excess pore pressure is larger than the weight and side friction at the upper part and accordingly water or gas is trapped in a thin gap between the upper part and the lower part [1]. Outburst is a kind of strong eruption of mixture of soils and water and gas [2].

If liquefiable or gasified sediment is overlain by a less permeable layer, then the overlying sediment (over-cap) can restrict pore water or gas to pass through. Once the pore pressure is larger than the weight and the side friction, the over-cap will be pushed upward to form a fracture or even crushed, which leads to a sudden unloading of pore pressure at the interface between the saturated or gasified sediment and the over-cap. Accordingly, an unloading wave will transmit into the sediment. Sediment can be fractured in layered form when the excess pressure is not too high; otherwise, the fracture failure becomes continuous and outburst occurs.

The main objective of this study is to investigate the mechanism and formation conditions of layered fracture and outburst in a soil layer. First, some typical experiments are introduced. Second, initiation conditions of these two types of failure forms are analyzed.

\section{LAYERED FRACTURE}

In general, layered fracture happens in a relatively low pore pressure. First, the over-cap is only pushed upward to form a gap by the pore fluid (gas or water or their mixture), and then one or more fractures occur in the liquefied or gasified zone if the pressure difference is high enough between the former fracture and the deeper zone. 
When the pressure $p_{0}$ in soil layer satisfies the condition

$$
p_{0} \geq p_{a}+\frac{\tau l}{A} L+\sigma_{t}
$$

layered fracture initiates, in which $p_{a}$ is the atmospheric pressure, $p_{0}$ is the pressure inside the soil, $\tau l / A$ is the side friction in unit length, and $\sigma_{t}$ is the tensile strength.

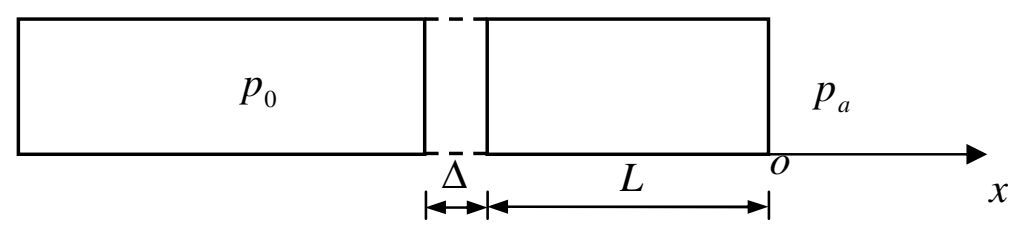

Fig. 1. Sketch of layered fracture

To analyze the movement of the layered zone, the following formulas are adopted:

$$
p-p_{a}-\frac{\tau l}{A} L=\rho_{s}\left(1-\varepsilon_{0}\right) L \cdot \ddot{\Delta}
$$

with initial condition

$$
t=0, \Delta=\Delta_{0}, \dot{\Delta}=0
$$

in which $p$ is the pressure in the fracture, $\rho_{s}$ is the density of soils, $\mathcal{E}_{0}$ is the initial porosity, $\Delta$ is the width of the fracture, and $\Delta_{0}$ is the initial width of the fracture (Fig. 1).

With the movement of the fracture zone, the fracture expands, and the pressure inside it decreases, causing the pore fluid to seep into the fracture from the two sides. To analyze the development of the fracture width, the following equations are adopted. The fracture zone is denoted by zone I, and the soil layer after the fracture is denoted by zone II.

In zone $\mathrm{I}$, the pressure $p_{1}$, density $\rho_{1}$, and velocity $u_{1}$ satisfy

$$
\left\{\begin{array}{c}
-\frac{\partial p_{1}}{\partial x}=\frac{\varepsilon_{0} \mu}{k} u_{1} \\
\frac{\partial \rho_{1}}{\partial t}+\frac{\partial \rho_{1} u_{1}}{\partial x}=0 \\
p_{1}=\rho_{1} a^{2}
\end{array}\right.
$$

with initial condition

$$
u_{1}=0, p_{1}=p_{0}
$$

and boundary condition

$$
x=-L, p_{1}=p ; x=0, p_{1}=p_{a}
$$


In zone II, the pressure $p_{2}$, density $\rho_{2}$, and velocity $u_{2}$ satisfy

$$
\left\{\begin{array}{c}
-\frac{\partial p_{2}}{\partial x}=\frac{\varepsilon_{0} \mu}{k} u_{2} \\
\frac{\partial \rho_{2}}{\partial t}+\frac{\partial \rho_{2} u_{2}}{\partial x}=0 \\
p_{2}=\rho_{2} a^{2}
\end{array}\right.
$$

with initial condition

and boundary condition

$$
u_{2}=0, p_{2}=p_{0}
$$

$$
x=-\infty, p_{2}=p_{0} ; x=-L, p_{2}=p
$$

in which $\mu$ is the viscosity of pore fluid, $k$ is the permeability, and $a$ is the sonic speed.

The total flow rate $Q$ from zone I and II into the fracture is

$$
Q=Q_{1}+Q_{2}=\varepsilon_{0} A\left(\rho_{1} u_{1}+\rho_{2} u_{2}\right)
$$

The mass and the density in the fracture are

$$
\begin{aligned}
& m=m_{0}+\int_{0}^{t} Q d t \\
& \rho=\frac{m}{A \cdot \Delta}
\end{aligned}
$$

in which $m_{0}$ is the initial mass of pore fluid in the fracture $\Delta_{0}$, and $m_{0}=\rho_{0} A \Delta_{0}$.

Using the formula $p=\rho a^{2}$, the relation between the pressure inside the fracture and the fracture width is

$$
p \cdot \Delta=a^{2}\left(\int_{0}^{t} \frac{Q}{A} d t+\frac{m_{0}}{A}\right)
$$

\section{OUTBURST}

Discontinuity theory is used here to analyze the initiation of outburst. The effective stress can be expressed as

$$
\sigma_{e}=p-\sigma
$$

in which $\sigma_{e}$ is the effective stress, $p$ is the pore pressure, and $\sigma$ is the total stress.

In general, during the unloading of the total stress, the effective stress $\sigma_{e}$ becomes gradually tensile. When the tensile stress reaches the maximum, the soil layer will include failure:

$$
p-\sigma=\sigma_{M}-2 v\left[\sigma_{s}-p\right]
$$


in which $\sigma_{M}$ is the tensile strength of soil layer at $p=0$ and $\sigma_{s}=0, v$ is the Poisson's ratio, and $\sigma_{s}$ is the lateral stress.

Assuming that outburst proceeds so fast that the relative movement between pore fluid and skeleton as well as the volume change of grains can be neglected, the relation between pressure and strain $E$ is given by

$$
p\left(\varepsilon_{0}+E\right)=p_{0} \varepsilon_{0}
$$

Before failure, the strain can be neglected, so that

$$
\sigma=\sigma_{D}=\alpha p_{0}-\sigma_{l}
$$

in which $\alpha=1-2 v, \sigma_{l}=\sigma_{M}-2 v \sigma_{s}$, and $\sigma_{l}$ is the tensile strength, considering the effects of lateral pressure.

Using the C-J condition as in the case of steady deflagration, the criterion of outburst can be obtained as

$$
p_{0}=\frac{2 p_{a}+\sigma_{l}+\sqrt{\left(2 p_{a}+\sigma_{l}\right)^{2}-4 \alpha p_{a}^{2}}}{2 \alpha}
$$

If the side friction $F_{s}$ is considered, then $\sigma_{D}$ in the above equations is instituted by

$$
\sigma_{D}^{*}=\sigma_{D}-F_{s}
$$

\section{CONCLUSIONS}

It is shown that once the pore pressure is large enough to cause the failure of overcap (forming a hole or being broken), layered fracture or outburst occurs in the liquefied or gasified sediments. Three critical pressures are present corresponding to the stable critical value, layer fracture critical value, and outburst critical value. The critical values are close to the values of the strength of sediments and the side friction.

\section{REFERENCES}

[1] Lu, X.B., Wu, Y.R., Zheng, Z.M.: Formation mechanism of cracks in saturated sand. Acta Mech. Sinica. 22(4), 377-383 (2006)

[2] Brennan, A.J., Madabhushi, S.P.G.: Liquefaction and drainage in stratified soil. ASCE J. Geotech. Geoenviron. Engrg. 131(7), 876-885 (2005)

[3] Yu, S.B., Cheng, C.M., Tan, Q.M., Ding, Y.S.: Damage of porous dedia containing pressurized gas by unloading and the maximum damage principle for critical outburst. Acta Mech. Sinica 29(6), 641-646 (1997) (in Chinese)

[4] Briaud, J.L., Chaouch, A.: Hydrate melting in soil around hot conductor. J. Geotech. and Geoenviron. Engrg. 123(7), 645-653 (1997) 